\title{
Expression of glycoprotein nonmetastatic melanoma protein $B$ in macrophages infiltrating injured mucosa is associated with the severity of experimental colitis in mice
}

\author{
FUMISATO SASAKI $^{1}$, KOTARO KUMAGAI ${ }^{1}$, HIROFUMI UTO ${ }^{1}$, YOICHIRO TAKAMI ${ }^{2}$, TAKESHI KURE ${ }^{1}$, \\ KAZUAKI TABU $^{1}$, YUICHRO NASU ${ }^{1}$, SHINICHI HASHIMOTO ${ }^{1}$, SHUJI KANMURA ${ }^{1}$, MASATSUGU NUMATA ${ }^{1}$, \\ AKIHIRO MORIUCHI $^{3}$, TOSHIO SAKIYAMA ${ }^{1}$, HIROHITO TSUBOUCHI ${ }^{3,4}$ and AKIO IDO ${ }^{1,3}$ \\ ${ }^{1}$ Digestive and Lifestyle Diseases, Department of Human and Environmental Sciences, Kagoshima University \\ Graduate School of Medical and Dental Sciences, Kagoshima 890-8544; ${ }^{2}$ Pharmaceutical \\ Care and Health Sciences, School of Pharmacy, Shujitsu University, Okayama 703-8516; \\ ${ }^{3}$ Department of HGF Tissue Repair and Regenerative Medicine, Kagoshima University \\ Graduate School of Medical and Dental Sciences, Kagoshima, 890-8544; \\ ${ }^{4}$ Kagoshima City Hospital, Kagoshima 890-8760, Japan
}

Received September 28, 2014; Accepted June 22, 2015

DOI: $10.3892 / \mathrm{mmr} .2015 .4408$

\begin{abstract}
Glycoprotein nonmetastatic melanoma protein B (Gpnmb) is a transmembrane glycoprotein, which negatively regulates the inflammatory responses of macrophages. However, the role of Gpnmb in intestinal macrophages remains to be fully elucidated. The present study aimed to investigate the expression of Gpnmb and its effects on colonic mucosal injuries associated with dextran sulfate sodium (DSS)-induced colitis in BALB/c mice, DBA/2J (D2) mice lacking Gpnmb and Gpnmb-transgenic DBA/2J mice (D2-gpnmb+). The colonic expression of Gpnmb increased with the severity of DSS-induced colitis in BALB/c mice, and macrophages infiltrating the inflamed mucosa were found to express Gpnmb. The D2 mice lacking Gpnmb exhibited more severe DSS-induced colitis, which was accompanied by higher levels of pro-inflammatory cytokines, including interleukin (IL)-1 $\beta$ and IL-6, compared with the D2-gpnmb+ mice. Following lipopolysaccharide stimulation, macrophages from the D2 mice expressed higher levels of pro-inflammatory cytokines and lower levels of IL-10, compared with the D2-gpnmb+mice. In addition, in the RAW264.7 murine macrophage cell line, knockdown of Gpnmb by small interfering RNA was associated with increased production of pro-inflammatory cytokines, which
\end{abstract}

Correspondence to: Dr Hirofumi Uto, Digestive and Lifestyle Diseases, Department of Human and Environmental Sciences, Kagoshima University Graduate School of Medical and Dental Sciences, 8-35-1 Sakuragaoka, Kagoshima 890-8544, Japan

E-mail: hirouto@m2.kufm.kagoshima-u.ac.jp

Key words: glycoprotein nonmetastatic melanoma protein B, experimental colitis, mucosal injury, pro-inflammatory cytokine were potentially mediated by the extracellular signal-regulated kinase (ERK) and p38 signaling pathways. The results of the present study indicated that macrophages infiltrating injured mucosa express Gpnmb, and that Gpnmb-positive macrophages may ameliorate inflammation in the intestinal mucosa by decreasing pro-inflammatory cytokine production via the ERK and p38 signaling pathways.

\section{Introduction}

Inflammatory bowel diseases (IBD), including ulcerative colitis and Crohn's disease, are intractable disorders of unknown etiology, which cause chronic inflammation of the gastrointestinal tract (1). A number of immune, genetic and environmental factors are known to affect the onset and progression of colitis $(2,3)$. Several studies have suggested that the innate immune system, which includes macrophages and dendritic cells, is involved in the pathogenesis of colitis (4-6).

Macrophages are often pro-inflammatory; infiltrating the injured mucosa and regulating the production of various inflammatory cytokines, including interleukin (IL)-1 $\beta$, IL-6, and tumor necrosis factor (TNF)- $\alpha$, as well as the neutrophil chemoattractant, IL-8. Levels of these cytokines are elevated in $\operatorname{IBD}(7,8)$ and animal models of colitis $(9,10)$. In addition, abnormally differentiated subsets of intestinal macrophages are key in T-helper (Th)1 cell-dominant chronic colitis, owing to elevated production of IL-12 and IL-23 (11). Consistent with thys, depletion of macrophages and dendritic cells has been observed to ameliorate disease symptoms in an IL-10 knockout mouse model of colitis (12). Previous studies have also demonstrated that alternatively activated M2 macrophages can ameliorate experimental colitis through increased production of IL-10 (13), and blood monocytes can differentiate into either anti-inflammatory macrophages or inflammatory dendritic cells in the colon, based on their 
microenvironment (14). Therefore, macrophages can adopt various phenotypes depending on the local environment (15); however, the precise mechanism underlying colitis remains to be fully elucidated.

Originally described in melanoma cells, glycoprotein nonmetastatic melanoma protein B (Gpnmb), also known as osteoactivin and DC-HIL, is a heavily N-glycosylated type I transmembrane domain protein, with a short cytoplasmic domain containing an endosomal-sorting motif (16-18). The gene encoding Gpnmb was isolated in our previous study, and was differentially expressed in the livers of rats fed a choline-deficient, L-amino acid-defined diet (19). In addition, our previous investigations demonstrated that transgenic Gpnmb expression attenuates the development of hepatic fibrosis (20). Previous studies have demonstrated that the expression of Gpnmb in macrophages functions as a feedback regulator of pro-inflammatory responses (21), and that the binding of Gpnmb on antigen-presenting cells to syndecan-4 on activated $\mathrm{T}$ cells inhibits $\mathrm{T}$-cell activation (22). Thus, the expression of Gpnmb on antigen-presenting cells may negatively regulate inflammation. However, the potential pathophysiological roles of Gpnmb in various disorders, including IBD, remain largely unknown.

The present study aimed to characterize Gpnmb-positive and -negative macrophages, and investigate their roles in injured colonic mucosa. In a murine model of experimental colitis, the effects of macrophages expressing and lacking Gpnmb on the injured colonic mucosa were examined. In addition, the effects on the expression of pro-inflammatory cytokines were examined in vivo and in vitro to determine whether Gpnmb-positive macrophages contribute to the severity of experimental colitis in mice.

\section{Materials and methods}

Animals. BALB/c mice ( $\mathrm{n}=30 ; 7-9$ weeks-old; male) were obtained from Kyudo Co., Ltd. (Tosu, Japan). DBA/2J Gpnmb mutant (D2) mice (7-9 weeks-old; 11 male and 14 female) and DBA/2J-gpnmb+ (D2-gpnmb+) mice (7-9 weeks-old, 11 male and 14 female), in which the mutated Gpnmb allele was replaced with a wild-type allele (23), were purchased from The Jackson Laboratory (Bar Harbor, ME, USA). The mice were housed in a $24^{\circ} \mathrm{C}$-controlled environment with a $12 \mathrm{~h}$ light/dark cycle, and two to three mice were housed together. The mice were allowed ad libitum access to standard mouse chow and tap water. All animal experiments were approved by the Institutional Animal Care and Use Committee of Kagoshima University (Kagoshima, Japan).

Dextran sulfate sodium (DSS)-induced colitis. To induce experimental colitis, the mice received 2 or $3 \%$ DSS (molecular weight, $50 \mathrm{kDa}$; Ensuiko Sugar Refining Co., Tokyo, Japan) in their drinking water for 7 or 5 days, respectively. The control mice received sterile filter-purified distilled water.

To evaluate the severity of colitis, the mice were weighed daily, and disease activity index (DAI) scores were calculated. DAI scores ranged between 0 and 4 , based on weight loss, stool consistency and the presence or absence of fecal blood (24). All mice were sacrificed with an overdose of sodium pentobarbital (65-95 mg/kg; Kyoritsu Seiyaku Co., Ltd., Tokyo, Japan) on days $0,2,5,7,10$, or 14 , and the large intestines were removed between the ileocecal junction and the anal verge. The large intestines were assessed for weight and length, incised longitudinally and fixed in 10\% buffered formalin (Wako Pure Chemical Industries, Ltd., Osaka, Japan). Intestinal inflammation was scored, as described previously (25). Briefly, grade 0 was afforded to normal colonic mucosa; grade 1 to colonic mucosa with loss of one-third of the crypts; grade 2 to colonic mucosa with loss of two-thirds of the crypts; grade 3 to colonic mucosa in which the lamina propria was covered with a single layer of epithelium and mild inflammatory cell infiltration was present; and grade 4 for colonic mucosa presenting erosions and marked inflammatory cell infiltration.

Cell culture. RAW264.7 murine macrophage cells were obtained from the Human Science Research Resources Bank (Osaka, Japan) and cultured $\left(2 \times 10^{6}\right.$ cells $\left./ \mathrm{ml}\right)$ in Dulbecco's modified Eagle's medium (DMEM; Sigma-Aldrich, St. Louis, MO, USA) supplemented with $10 \%$ fetal bovine serum (FBS; Invitrogen Life Technologies, Carlsbad, CA, USA). The RAW264.7 cells were treated with $100 \mu \mathrm{g} / \mathrm{ml}$ lipopolysaccharide (LPS; Sigma-Aldrich) or phosphate-buffered saline (PBS) for $24 \mathrm{~h}$ at $37^{\circ} \mathrm{C}$.

In order to inhibit the expression of Gpnmb in the RAW264.7 cells, small interfering (si)RNA specific for Gpnmb (5'-UUAGCAUCUUCCUUCUGGCAUCUGG-3'; Invitrogen Life Technologies) was transfected into the cells using RNAiMAX (Invitrogen Life Technologies). Stealth RNAi Negative Control Medium GC Duplex (Invitrogen Life Technologies) was used as a negative control siRNA (NC-siRNA). One day prior to transfection, the cells were plated in $500 \mu$ 1 DMEM without antibiotics. The cells were $30-50 \%$ confluent at the point of transfection. The macrophages transfected with siRNA were cultured in RPMI-1640 medium (Invitrogen Life Technologies) supplemented with $10 \% \mathrm{FBS}$ for $24 \mathrm{~h}$ at $37^{\circ} \mathrm{C}$ in a $\mathrm{CO}_{2}$ incubator.

Isolation of thioglycollate-elicited peritoneal macrophages (TEPMs). The D2 and D2-gpnmb+ mice were intraperitoneally injected with $1 \mathrm{ml} \mathrm{10 \%} \mathrm{thioglycollate} \mathrm{(Sigma-Aldrich).}$ The TEPMs were harvested via peritoneal lavage with PBS 3 days post-injection, and were cultured in RPMI-1640 medium supplemented with $10 \%$ FBS. Following a $24 \mathrm{~h}$ incubation at $37^{\circ} \mathrm{C}$, the medium was replaced, and the TEPMs were treated with $10 \mu \mathrm{g} / \mathrm{ml}$ LPS or PBS for an additional $24 \mathrm{~h}$.

$R N A$ extraction, and reverse transcription-quantitative polymerase chain reaction ( $R T-q P C R)$. RNA was extracted from the cells or tissues following homogenization using TRIzol ${ }^{\circledR}$ regent (Invitrogen Life Technologies). Total RNA $(0.5 \mu \mathrm{g})$ was reverse transcribed using a PrimeScript ${ }^{\mathrm{TM}}$ RT Reagent kit (Takara Bio Inc., Otsu, Japan). Each reaction mixture for RT (total volume $20 \mu \mathrm{l}$ ) consisted of total RNA (adjusted to $500 \mathrm{ng}$, $2.5 \mu \mathrm{l})$, oligo dT PCR primer (50 $\mu \mathrm{M}, 1 \mu \mathrm{l}), 5 \mathrm{X}$ PrimeScript buffer $(4 \mu \mathrm{l})$, PrimeScript RT enzyme mix $(1 \mu \mathrm{l})$, random 6 mers $(100 \mu \mathrm{M}, 1 \mu \mathrm{l})$ and RNase free distilled $\mathrm{H}_{2} \mathrm{O}(10.5 \mu \mathrm{l})$. The synthesized cDNA was amplified by RT-qPCR using SYBR Premix Ex Taq II (Takara Bio Inc.). Each reaction mixture for PCR (total volume $20 \mu \mathrm{l}$ ) consisted of cDNA (adjusted to $500 \mathrm{ng}, 2 \mu \mathrm{l})$, forward and reverse PCR primer $(10 \mu \mathrm{M}, 0.8 \mu \mathrm{l}$ 
Table I. Polymerase chain reaction primer sets.

\begin{tabular}{lll}
\hline Gene & \multicolumn{1}{c}{ Forward primer $\left(5^{\prime}-3^{\prime}\right)$} & \multicolumn{1}{c}{ Reverse primer $\left(5^{\prime}-3^{\prime}\right)$} \\
\hline Gpnmb & ATCCCTGGCAAAGACCCAGAC & CCTATTGGCTTGTACGCCTTGTG \\
IL- $\beta$ & TCCAGGATGAGGACATGAGCAC & GAACGTCACACACCAGCAGGTTA \\
IL-6 & CCACTTCACAAGTCGGAGGCTTA & GCAAGTGCATCATCGTTGTTCATAC \\
TNF- $\alpha$ & AAGCCTGTAGCCCACGTCGTA & GGCACCACTAGTTGGTTGTCTTTG \\
MCP- 1 & GCATCCACGTGTTGGCTCA & CTCCAGCCTACTCATTGGGATCA \\
$\beta$-actin & CATCCGTAAAGACCTCTATGCCAAC & ATGGAGCCACCGATCCACA
\end{tabular}

Gpnmb, glycoprotein nonmetastatic melanoma protein B, IL, interleukin, TNF- $\alpha$, tumor necrosis factor- $\alpha$; MCP-1, monocyte chemoattractant protein-1.

each), SYBR Premix Ex Taq II (10X, $10 \mu \mathrm{l})$, ROX reference dye $(50 \mathrm{X}, 0.4 \mu \mathrm{l})$ and distilled $\mathrm{H}_{2} \mathrm{O}(6 \mu \mathrm{l})$. The synthesized cDNA was quantified using an ABI prism 7700 Sequence Detector (Applied Biosystems Life Technologies, Foster City, CA, USA). The cycling conditions were as follows: One cycle at $95^{\circ} \mathrm{C}$ for $30 \mathrm{sec}$ followed by 35 cycles each at $95^{\circ} \mathrm{C}$ for $5 \mathrm{sec}$ and $60^{\circ} \mathrm{C}$ for $34 \mathrm{sec}$. The PCR primer sequences used are listed in Table I (Sigma-Aldrich). Gene expression levels were normalized to those of $\beta$-actin, which was used as an internal control.

Western blot analysis. The RAW264.7 cells and TEPM from the D2 or D2-gpnmb+ were also washed PBS and removed from the dishes using a cell scraper with $0.5 \%$ SDS (Promega Corporation, Madison, WI, USA). The cell lysates were heated to $100^{\circ} \mathrm{C}$ for $5 \mathrm{~min}$ and cooled on ice. Following centrifugation at $450 \mathrm{x} \mathrm{g}$ for $5 \mathrm{~min}$ at $20^{\circ} \mathrm{C}$, the cell lysates were analyzed for protein content using a Lowry Assay (Bio-Rad Laboratories Inc., Hercules, CA, USA). Equal quantities of protein $(1 \mu \mathrm{g} / \mu \mathrm{l})$ were separated on 10\% SDS-PAGE (Wako Pure Chemical Industries, Ltd.) and transferred onto polyvinylidene fluoride membranes (Bio-Rad Laboratories, Inc.). Following blocking overnight at $4^{\circ} \mathrm{C}$ with $5 \%$ non-fat milk, the blots were probed with primary antibodies for $1 \mathrm{~h}$ at room temperature. The membranes were then incubated with the following primary antibodies: Polyclonal goat anti-mouse Gpnmb (1:1,000; cat. no. AF2330) purchased from R\&D systems, Inc. (Minneapolis, MN, USA). Polyclonal rabbit anti-mouse phosphorylated (p)-ERK1/2 (1:1,000, cat. no. 9101), ERK1/2 (1:1,000, cat. no. 3372), phosphorylated c-jun N-terminal kinase (p-JNK; 1:1,000; cat. no. 9251), p-p mitogen activated kinase (MAPK; 1:1,000; cat. no. 4511), p38 MAPL (1:1,000, cat. no. 9212) and IкB (1:1,000; cat. no. 9242), as well as monoclonal rabbit anti-mouse JNK (1:1,000; cat. no. 9258) were purchased from Cell Signaling Technology Inc. (Danvers, MA, USA). Monoclonal mouse anti- $\beta$-actin (1:1,000; cat. no. A5441) was purchased from Sigma-Aldrich. The membranes were washed with Tris-buffered saline (Bio-Rad Laboratories, Inc.) with Tween 20 (Wako Pure Chemical Industries) three times. Following incubation of the membranes with the appropriate peroxidase-conjugated secondary antibodies [polyclonal donkey anti-goat immunoglobulin G-horseradish peroxidase (IgG-HRP), 1:2,000, cat. no. sc-2033; polyclonal goat anti-rabbit IgG-HRP, 1:2,000, cat. no. sc-2004] purchased from Santa Cruz Biotechnology, Inc. (Dallas, TX, USA) for $1 \mathrm{~h}$ at room temperature, the membranes were visualized using an Enhanced Chemiluminescence Detection kit (cat. no. RPN2232 GE Heathcare, Buckinghamshire, UK). Secondary antibodies were diluted using Can Get Signal ${ }^{\circledR}$ (cat. no. NKB-301; Toyobo Co., Ltd., Osaka, Japan) Expression level quantification was performed using Image J version 1.48 (National Institutes of Health, Bethesda, MD, USA), and $\beta$-actin was used as an internal control.

Immunohistochemistry. For immunohistochemical analysis, the colon tissues were fixed in $10 \%$ buffered formalin and incubated with goat anti-mouse Gpnmb antibodies (R\&D Systems, Inc.) or rat anti-mouse F4/80 antibodies (AbD Serotec, Kidlington, UK). For immunofluorescence analysis, paraffin-embedded (Sigma-Aldrich) and $2 \mu \mathrm{m}$ frozen tissue sections were incubated with goat anti-mouse Gpnmb antibodies (R\&D Systems, Inc.) or rat anti-mouse CD68 antibodies (AbD Serotec), followed by imaging under a fluorescence microscope (BZ-9000; Keyence Corporation, Osaka, Japan).

ELISA. The concentrations of IL-1 $\beta$, IL-6, TNF- $\alpha$, MCP-1, and IL-10 were measured in the culture supernatants using the following cytokine ELISA kits; IL- $\beta$ mouse ELISA (Invitrogen Life Technologies), mouse IL-6 quantikine ELISA (R\&D Systems, Inc.), mouse IL-10 quantikine ELISA (R\&D Systems, Inc.), mouse TNF- $\alpha$ quantikine ELISA (R\&D Systems, Inc.), and MCP-1 mouse ELISA (Invitrogen Life Technologies). Samples were analyzed in duplicate using a microplate reader model 680 (Bio-Rad Laboratories, Inc.). The concentrations of cytokines in the samples were calculated using standard curves. Protein concentrations in each sample were determined using an RC DC protein assay (Bio-Rad Laboratories, Inc.).

Statistical analysis. Statistical analysis was performed using SPSS software version 17.0 (SPSS, Inc., Chicago, IL, USA). Data were analyzed using the Mann-Whitney U test or Tukey's test. $\mathrm{P}<0.05$ was considered to indicate a statistically significant difference.

\section{Results}

Gpnmb is expressed in macrophages infiltrating into injured colonic mucosa. Following administration of the mice with 
A

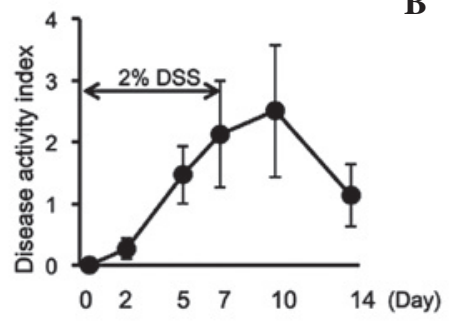

C

HE

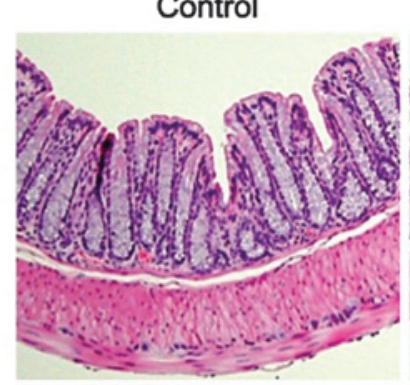

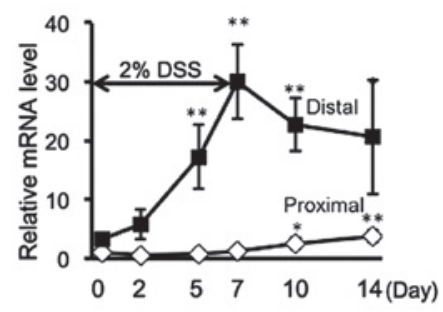

$2 \%$ DSS

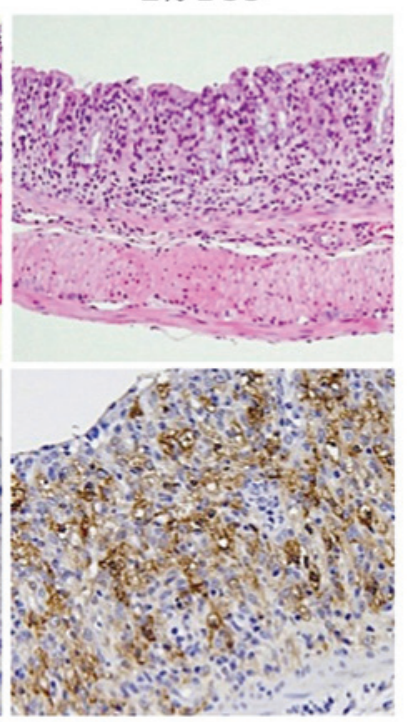

D
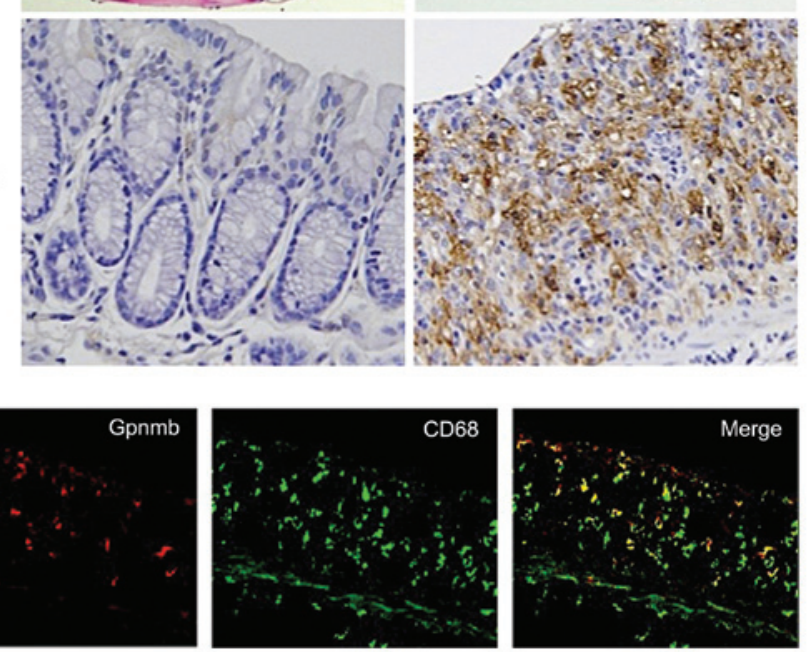

Figure 1. Expression of Gpnmb in the injured colonic mucosa of mice with DSS-induced colitis. (A) Disease activity index scores were evaluated for 14 days in mice with DSS-induced colitis. Data are presented as the mean $\pm \mathrm{SD}(\mathrm{n}=5)$. (B) mRNA expression levels of Gpnmb in the proximal and distal colon tissues from mice with DSS-induced colitis were examined using reverse transcription-quantitative polymerase chain reaction. The expression of Gpnmb gradually increased following the administration of DSS and remained elevated until day 14, despite withdrawal of DSS at day 7. mRNA expression levels of Gpnmb in the proximal colon tissues at day 0 were set as 1 . Data are presented as the mean $\pm \mathrm{SD}(\mathrm{n}=5){ }^{*} \mathrm{P}<0.01$ and ${ }^{* *} \mathrm{P}<0.001$, vs. day 0 . (C) Histological analysis of distal colon tissues from mice treated with vehicle (control) or $2 \%$ DSS for 7 days. Representative images of HE staining and immunohistochemistry for Gpnmb are shown at original magnifications of 40x and 100x, respectively. (D) Dual-immunofluorescence analysis of Gpnmb and CD68 in distal colon tissues of mice treated with $2 \%$ DSS for 7 days, shown at the original magnification of 100x. Gpnmb, glycoprotein nonmetastatic melanoma protein B; DSS, dextran sulfate sodium; HE, hematoxylin and eosin; SD, standard deviation.

$2 \%$ DSS for 7 days, DAI scores gradually increased until day 10 (3 days following removal of DSS), and remained elevated above baseline until day 14 (Fig. 1A). mRNA expression levels of Gpnmb were increased in parallel with the development of colitis in the distal large intestine (Fig. 1B), which was the primary site of DSS-induced mucosal injury. The mRNA expression levels peaked on day 7 and remained elevated even following the cessation of DSS treatment (Fig. 1B). By contrast, the mRNA expression levels of Gpnmb remained low in the proximal large intestine throughout the experimental period (14 days; Fig. 1B). Furthermore, protein expression of Gpnmb was confirmed on day 7 using western blot analysis, which was observed in the distal large intestine, but not in the proximal large intestine (data not shown). Histologically, the mice treated with $2 \%$ DSS exhibited destruction of epithelial tissue, infiltration of inflammatory cells into the mucosa and submucosa, edema and mucosal thickening by day 7 (Fig. 1C). Gpnmb was also observed in the infiltrating inflammatory cells, but not in epithelial cells, determined using immunohistochemistry (Fig. 1C). In order to detect the cells positively expressing Gpnmb, double immunostaining was performed on the samples. Gpnmb was expressed in a subset of cells positive for the macrophage marker CD68 (Fig. 1D), and also in a subset of cells positive for F4/80 (data not shown). These results indicated that Gpnmb is expressed in macrophages infiltrating into the injured colonic mucosa.

DSS-induced mucosal injury is exacerbated in mice lacking Gpnmb. DSS (3\%) was administered for 5 days to D2 mice, which exhibit a naturally occurring mutation in Gpnmb 
A

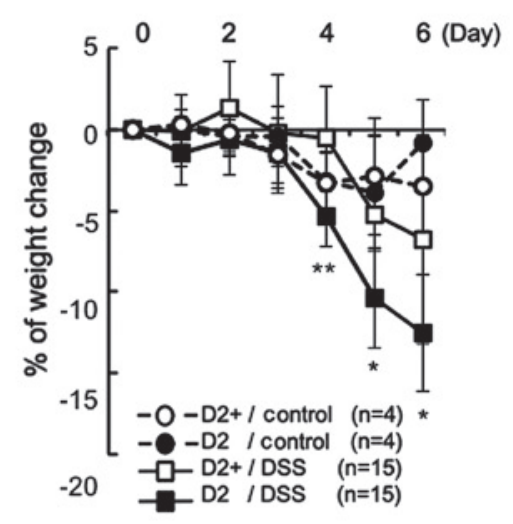

C

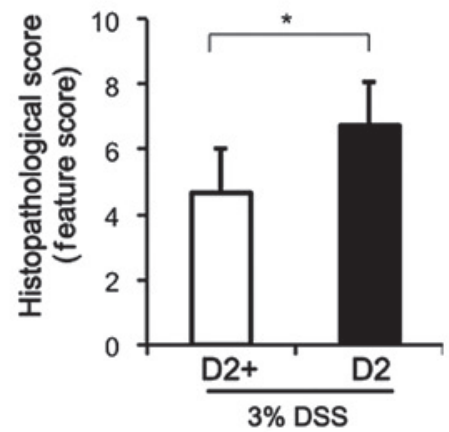

$\mathbf{E}$

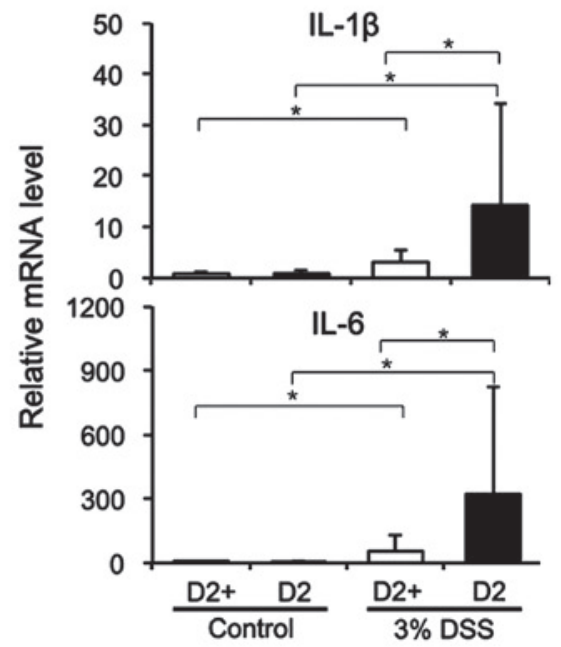

B

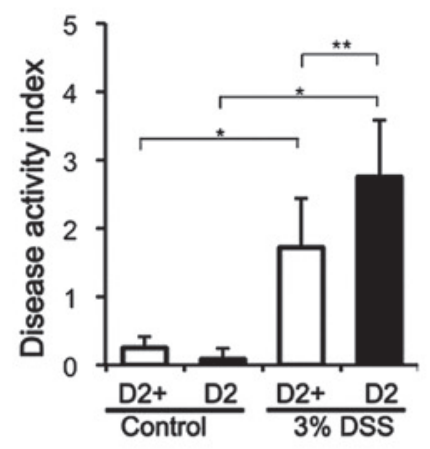

D
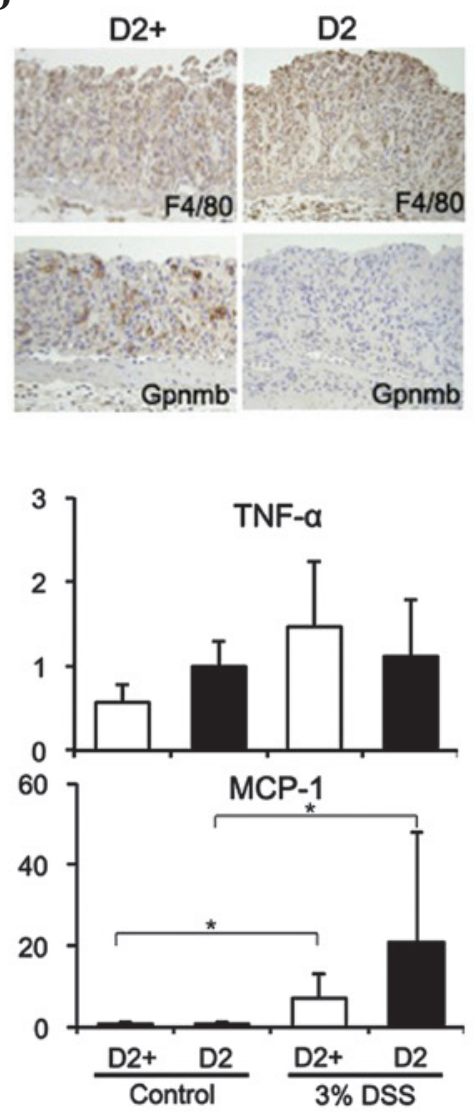

Figure 2. Gpnmb ameliorates DSS-induced colitis and expression of pro-inflammatory cytokines in colon tissues. D2 mice lacking Gpnmb and D2-gpnmb+ (D2+) mice expressing wild-type Gpnmb were treated with 3\% DSS or water for 5 days. (A) Changes in body weight (\% weight change) and (B) disease activity index scores were examined on day 6. Data are presented as the mean $\pm \mathrm{SD}(\mathrm{n}=5) .{ }^{*} \mathrm{P}<0.01 ;{ }^{* * *} \mathrm{P}<0.001$. (C) Histological scores for the $\mathrm{D} 2$ and $\mathrm{D} 2+$ mice treated with 3\% DSS for 5 days. Data are presented as the mean $\pm \mathrm{SD}(\mathrm{n}=5)$. ${ }^{*} \mathrm{P}<0.001$. (D) Immunohistochemical analysis of F4/80 and Gpnmb in colon tissues of D2 and D2+ mice administered 3\% DSS for 5 days. Representative images are shown. (E) mRNA expression levels of IL-1 $\beta$, IL-6, TNF- $\alpha$ and MCP-1 were detected in colon tissues using reverse transcription-quantitative polymerase chain reaction. mRNA expression levels in the colon tissues of D2+ mice treated with water were set as 1 . Data are presented as the mean $\pm \mathrm{SD}(\mathrm{n}=5) .{ }^{*} \mathrm{P}<0.01$. Gpnmb, glycoprotein nonmetastatic melanoma protein $\mathrm{B}$; DSS, dextran sulfate sodium; SD, standard deviation; IL, interleukin; TNF, tumor necrosis factor; MCP, monocyte chemoattractant protein.

(R150X), and to D2-gpnmb+ mice, in which the mutated Gpnmb allele was replaced with a wild-type allele (21). The body weight of the $\mathrm{D} 2$ mice decreased significantly as colitis developed, compared with the D2-gpnmb+ mice treated with DSS $(\mathrm{P}<0.001$ on day $4 ; \mathrm{P}<0.01$ on days 5 and 6$)$, as shown in Fig. 2A, and the D2 mice had significantly higher DAI scores on day 5 ( $\mathrm{P}<0.001$; Fig. 2B). The colonic weight following administration of DSS for 5 days was similar in the $\mathrm{D} 2$ and $\mathrm{D} 2$-gpnmb+ mice; however, the colon lengths were significantly shorter in the DSS-treated D2 mice, compared with the $\mathrm{D} 2$-gpnmb+ mice $(7.1 \pm 0.8$, vs. $8.0 \pm 1.1 \mathrm{~cm} ; \mathrm{P}<0.01)$. Histological analyses revealed that colon tissues from the DSS-treated D2-gpnmb+ mice were characterized by shorter crypts and mild inflammatory cell infiltration, with no epithelial cell destruction. By contrast, DSS-induced colitis in the D2 mice resulted in complete loss of crypts, destruction of the epithelium and severe inflammatory cell infiltration in the colon tissues (data not shown). In addition, histopathological 
A

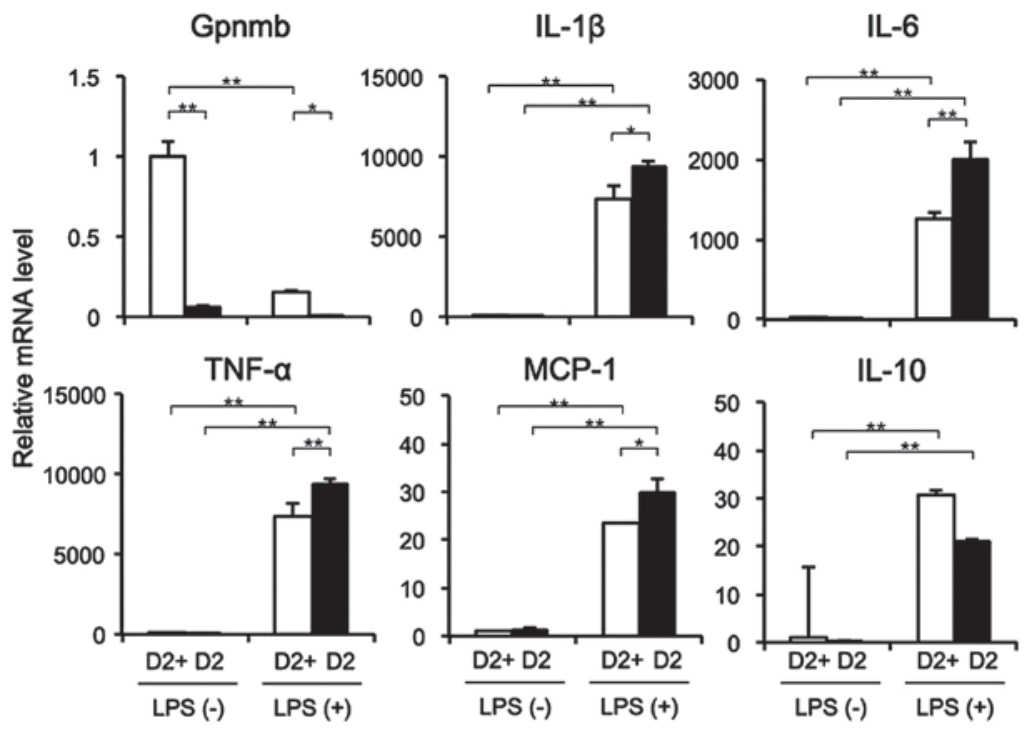

B
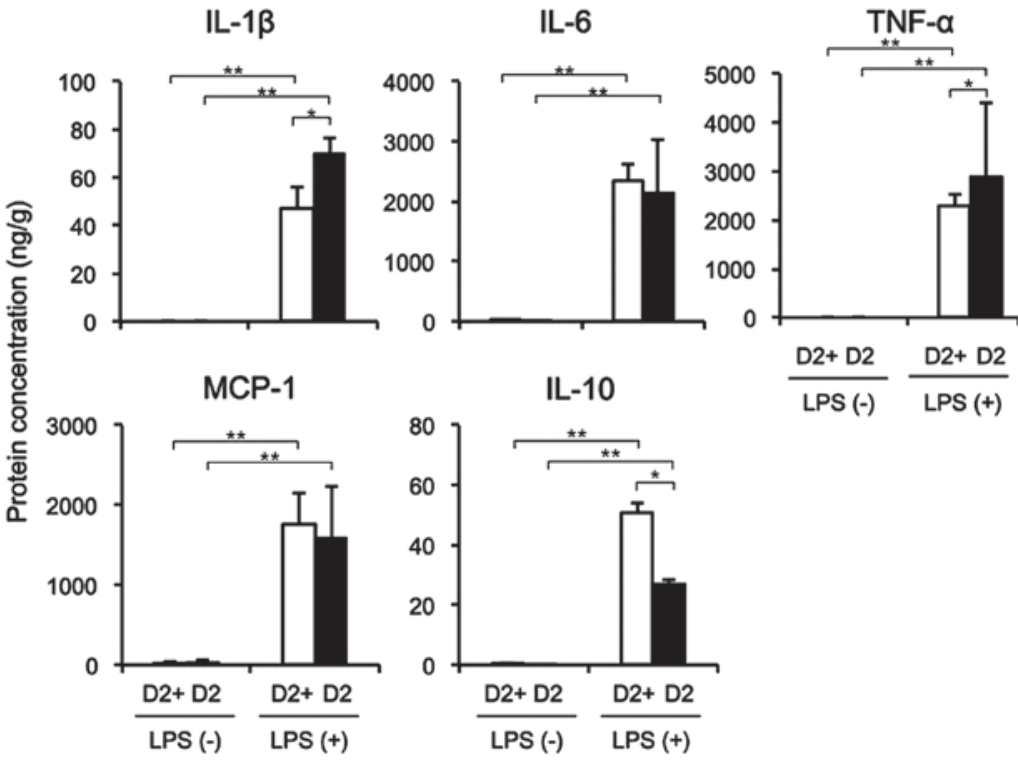

Figure 3. TEPMs lacking Gpnmb expression exhibit enhanced expression of pro-inflammatory cytokines. TEPMs were isolated from D2 and D2-gpnmb+ (D2+) mice. (A) mRNA expression levels of Gpnmb, IL-1 $\beta$, IL-6, TNF- $\alpha$, MCP-1 and IL-10 in the absence or presence of LPS were detected using reverse transcription-quantitative polymerase chain reaction. mRNA expression levels in TEPMs isolated from D2+ mice without LPS treatment were set as 1. (B) Protein levels of IL-1 $\beta$, IL-6, TNF- $\alpha$, MCP-1 and IL-10 in the supernatants of TEPMs in the absence or presence of LPS, examined using ELISA. The protein levels were adjusted by the total protein concentration in the supernatant. Data are presented as the mean \pm standard deviation ( $n=3$ ). "P<0.01; ${ }^{* *} \mathrm{P}<0.001$. TEMPs, thioglycollate-elicited peritoneal macrophages; Gpnmb, glycoprotein nonmetastatic melanoma protein B; DSS, dextran sulfate sodium; IL, interleukin; TNF, tumor necrosis factor; MCP, monocyte chemoattractant protein; LPS, lipopolysaccharide.

scoring revealed that DSS-induced colitis in the D2 mice was significantly exacerbated, compared with that in the D2-gpnmb+ mice (P<0.05; Fig. 2C).

The expression of Gpnmb was observed in the distal colonic tissues from the DSS-treated D2-gpnmb+ mice (Fig. 2D), however, no Gpnmb was observed in the colon tissues isolated from the DSS-treated D2 mice. In addition, marked infiltration of F4/80-positive macrophages into the injured colonic mucosa was observed in the D2-gpnmb+ and D2 mice, and a number of macrophages from the D2-gpnmb+ mice expressed Gpnmb (Fig. 2D). As expected, the expression of Gpnmb was not detected in macrophages infiltrating the injured colonic mucosa of the D2 mice, regardless of whether DSS was administered.
When the D2 and D2-gpnmb+ mice were treated with $3 \%$ DSS for 5 days, the mRNA expression levels of IL-1 $\beta$, IL-6, and MCP-1 were significantly elevated, and significantly higher levels of IL-1 $\beta$ and IL- 6 were observed in the injured colon tissues from the D2 mice, compared with in those from the D2-gpnmb+ mice (Fig. 2E). The D2 mice treated with 3\% DSS also exhibited elevated expression levels of MCP-1 in injured colon tissues; however, no significant difference was observed in the expression of MCP-1 between the D2 and $\mathrm{D} 2$-gpnmb+ mice ( $\mathrm{P}=0.075$; Fig. 2E).

TEPMs lacking Gpnmb expression exhibit enhanced expression of pro-inflammatory cytokines. The mRNA expression of Gpnmb in the TEPMs from the D2 mice was low or 
A

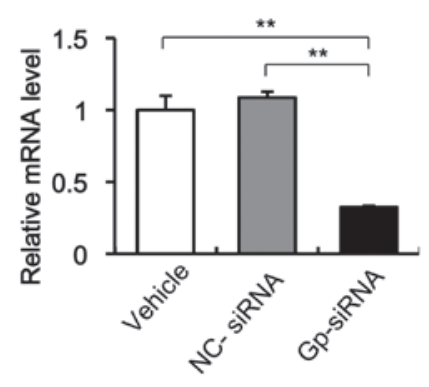

C
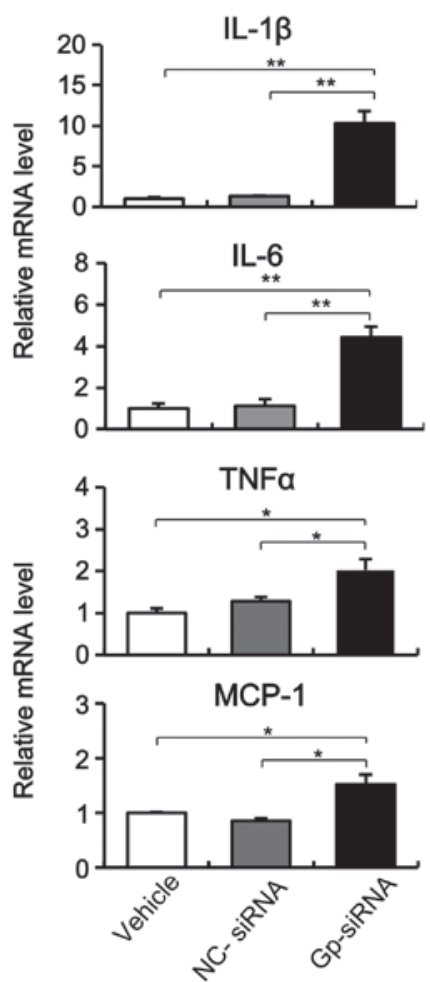

B
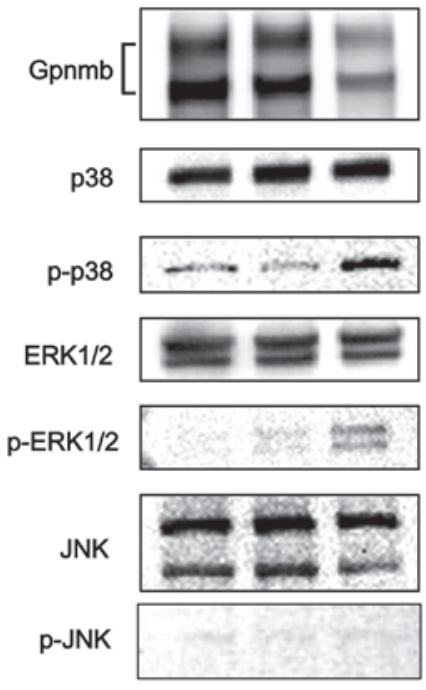

IKB

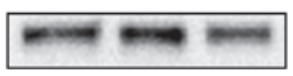

$\beta$-actin

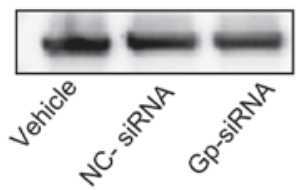

Gpnmb

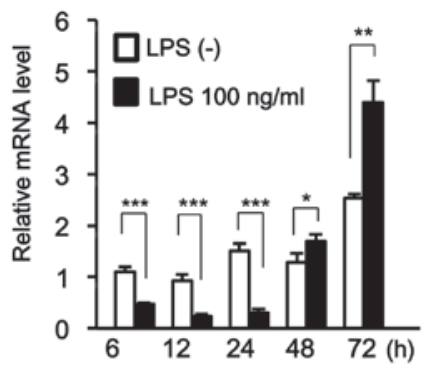

Figure 4. Reduced Gpnmb expression increases cytokine and chemokine production and activates p38 and ERK1/2 signaling. (A) RAW264.7 murine macrophage cells were transfected with NC-siRNA, Gp-siRNA, or vehicle alone, and the expression of Gpnmb was examined using RT-qPCR. Data are presented as the mean $\pm \mathrm{SD}\left(\mathrm{n}=3\right.$; $\left.{ }^{*} \mathrm{P}<0.001\right)$. (B) Effects of Gpnmb knockdown on levels of mRNAs encoding IL-1 $\beta$, IL-6, TNF- $\alpha$ and MCP-1 were examined using RT-PCR. Data are presented as the mean $\pm \mathrm{SD}\left(\mathrm{n}=3 ;{ }^{*} \mathrm{P}<0.01 ;{ }^{* *} \mathrm{P}<0.001\right.$. (C) Effects of Gpnmb knockdown on p38, ERK1/2, and JNK signaling and I $\mathrm{B}$ activation were examined using western blotting. (D) Expression of Gpnmb in the RAW264.7 cells was decreased in response to LPS and increased following the removal of LPS. RAW264.7 macrophage cells were exposed to LPS (100 $\mu \mathrm{g} / \mathrm{ml})$ for $24 \mathrm{~h}$ and changes in Gpnmb were examined using RT-qPCR. mRNA expression levels in the cells without LPS for $6 \mathrm{~h}$ were set as 1 . Data are presented as the mean $\pm \mathrm{SD}(\mathrm{n}=3)$. ${ }^{*} \mathrm{P}<0.05 ;{ }^{* *} \mathrm{P}<0.01 ;{ }^{* * *} \mathrm{P}<0.001$. Gpnmb, glycoprotein nonmetastatic melanoma protein B; IL, interleukin; TNF, tumor necrosis factor; MCP, monocyte chemoattractant protein; LPS, lipopolysaccharide; ERK, extracellular signal-regulated kinase; NC, negative control; Gp, Gpnmb; siRNA, small interfering RNA; RT-qPCR, reverse transcription-quantitative polymerase chain reaction; JNK, c-Jun N-terminal kinase; p-, phosphorylated.

undetectable, regardless of LPS stimulation; whereas the mRNA expression of Gpnmb was reduced in the D2-gpnmb+ mice following LPS stimulation (Fig. 3A). In the absence of LPS, TEPMs from the D2-gpnmb+ and D2 mice expressed low mRNA levels of IL-1 $\beta$, IL- 6 , TNF- $\alpha$, MCP-1 and IL-10. Conversely, LPS stimulated the expression of these mRNAs in the TEPMs isolated from the D2-gpnmb+ and D2 mice, and the mRNA expression levels of IL- $1 \beta$, IL-6, TNF- $\alpha$ and MCP-1 were significantly higher in the TEPMs from D2 mice, compared with those from the D2-gpnmb+ mice. In addition, the expression of IL-10 was markedly lower following LPS stimulation in the D2 mice, compared with the D2-gpnmb+ mice, although this difference was not statistically significant.
The protein expression levels of IL- $1 \beta$ and TNF- $\alpha$ were significantly higher, and the expression of IL-10 was significantly lower in the supernatants of TEPMs from the D2 mice, compared with those from the D2-gpnmb+ mice (Fig. 3B). These results suggested that the inhibition of inflammatory cytokines and the stimulation of anti-inflammatory cytokines by Gpnmb in macrophages may reduce inflammation in the injured colonic mucosa.

Expression of Gpnmb in response to LPS, and its effects on cytokine and chemokine production in murine macrophages. The siRNA-mediated knockdown of Gpnmb in RAW264.7 macrophages in the present study was effective. 
The Gpnmb-specific siRNA (Gp-siRNA) reduced the mRNA and protein expression levels of Gpnmb to $\sim 1 / 3$ of the levels observed in the cells transfected with NC-siRNA (Fig. 4A and B). Furthermore, inhibition of the expression of Gpnmb led to significant increases in the expression levels of IL-1 $\beta$, IL-6, TNF- $\alpha$ and MCP-1 (Fig. 4C). In addition, when the expression of Gpnmb was knocked down by Gp-siRNA, p38 and ERK1/2 were phosphorylated at significantly higher levels; whereas the phosphorylation of JNK and the expression of IкB were not significantly affected (Fig. 4C). These results indicated that Gpnmb negatively regulated the production of pro-inflammatory cytokines and chemokines by inhibiting signaling pathways involving p38 and ERK1/2.

Macrophages infiltrating into injured tissues are have been suggested to alter phenotypes in response to the myriad of stimuli present during wound healing (26). The present study investigated changes in the expression of Gpnmb in murine RAW264.7 cells in response to LPS. The RAW264.7 cells expressed Gpnmb in the absence of LPS (Fig. 4B). The cells exposed to LPS for $24 \mathrm{~h}$ expressed significantly lower levels of Gpnmb during the treatment period, compared with the control cells treated with PBS alone. However, the expression of Gpnmb increased significantly over the 2 days following the removal of LPS (Fig. 4D).

\section{Discussion}

The results of the present study demonstrated that DSS induced mucosal injury between the rectum and the distal colon, whereas the proximal colon remained unaffected. In this murine model, infiltrating macrophages expressed Gpnmb in injured colonic tissues, whereas the expression of Gpnmb was almost undetectable in the proximal colon tissues, even when the mice were treated with DSS for 7 days. These results indicated that macrophages recruited to the injured mucosal tissue express Gpnmb, whereas the resident macrophages of the uninjured intestine do not. In addition, the results suggested a potential role for Gpnmb in suppressing inflammatory responses in colitis by attenuating pro-inflammatory cytokine production mediated by $\mathrm{p} 38$ and ERK signaling.

An inflammatory environment causes recruited macrophages to assume heterogeneous phenotypes, which together assist in balancing the damaging and healing mechanisms within the tissue $(14,27)$. The present study demonstrated that the expression of Gpnmb gradually increased during 7 days of DSS administration, and remained elevated, even following the removal of DSS from the drinking water. In addition, when RAW264. 7 cells were treated with LPS for $24 \mathrm{~h}$, the expression of Gpnmb was reduced in response to inflammatory stimuli in vitro; however, the expression of Gpnmb gradually increased following removal of the LPS, ultimately rising above the baseline level at $72 \mathrm{~h}$. These results suggested that macrophages infiltrating the injured mucosa expressed Gpnmb in response to the inflammatory environment, and that these Gpnmb-positive macrophages were involved in a delayed phase of inflammation and/or tissue repair processes.

Macrophages involved in tissue repair can assume the alternatively activated phenotype (26), whereas macrophages, which are isolated from wounds, are not alternatively activated. The phenotype of macrophages in injury changes over time, and does not reflect the classic classification schemes (28). In the present study, the expression of Gpnmb in the macrophage cell line was downregulated in the presence of LPS, but was upregulated following the removal of LPS. In vivo experiments demonstrated that elevated levels of Gpnmb persisted following the removal of DSS from the drinking water. These results suggested that Gpnmb-expressing macrophages are involved in the wound-healing process and ameliorate mucosal inflammation by modulating the expression of pro-inflammatory cytokines.

In the identification of genes, which are preferentially expressed in macrophages, Rippoll et al (21) identified Gpnmb as a macrophage-specific gene, reporting that the expression of Gpnmb is inhibited in RAW264.7 cells exposed to LPS for $21 \mathrm{~h}$. Conversely, the expression of Gpnmb in RAW264.7 cells reduces the LPS/interferon (IFN)- $\gamma$-induced secretion of IL-6 and IL-12p40 (21). The results of the present study were consistent with these findings; in which the expression of Gpnmb in RAW264.7 cells decreased during $24 \mathrm{~h}$ treatment with LPS. Furthermore, siRNA-mediated knockdown of Gpnmb in RAW264.7 cells increased the expression of IL-1 $\beta$, IL-6, TNF- $\alpha$ and MCP-1, in the absence of LPS and IFN- $\gamma$. These results indicated that Gpnmb suppressed pro-inflammatory cytokine production in macrophages, suggesting that Gpnmb-positive macrophages negatively regulates inflammation in the injured intestinal mucosa. In addition, the present study demonstrated that inhibition of the expression of Gpnmb in RAW264.7 cells resulted in the enhanced phosphorylation of p38 and ERK1/2; however, no significant difference was observed in the expression of I $\mathrm{KB}$, which is directly associated with nuclear factor (NF)-KB activation. Zhou et al (29) previously demonstrated that the absence of Gpnmb increased the expression of IL-18 in the iris/ciliary body by increasing the activation of NF- $\mathrm{KB}$ and the phosphorylation of MAPKs, predominantly in CD69-positive cells. These results suggest that Gpnmb suppresses pro-inflammatory cytokine production in inflammatory cells via the attenuation of MAPK signaling. Furthermore, p38/MAPK phosphatase-1 regulates AKT, which coordinates phenotypic transitions of macrophages, and resolution of inflammation during tissue repair (30). Akt1 and Akt2 also contribute differentially to macrophage polarization (31). Therefore, Gpnmb may be associated with macrophage polarization.

In conclusion, the present study demonstrated that macrophages infiltrating the injured intestinal mucosa express Gpnmb. These cells are anti-inflammatory, due to their decreased production of pro-inflammatory cytokines, and elevated levels of Gpnmb persisted following removal of the inflammatory stimulus. These results suggested that Gpnmb-positive macrophages may be important in ameliorating mucosal injury. Although further investigations are required to clarify the roles of Gpnmb-positive macrophages in the injured mucosa, these findings provide novel information regarding the roles of innate immunity in tissue injury, inflammation and healing of the intestinal mucosa.

\section{Acknowledgements}

The authors would like to thank Ms. Yuko Morinaga-Nakamura for her technical assistance. This study was supported by grants 
from the Ministry of Education, Culture, Sports, Science and Technology of Japan (grant nos. 19590763 and 22590742 to Dr Akio Ido), and from the Ministry of Health, Labour and Welfare of Japan as an official project of the Intractable Inflammatory Bowel Diseases Study Group of Japan (to Dr Hirohito Tsubouchi) Drs Akihiro Moriuchi, Hirohito Tsubouchi and Dr Akio Ido hold endowed faculty positions in research for HGF tissue repair and regenerative medicine, and received funds from Eisai Co., Ltd (Tokyo, Japan).

\section{References}

1. Podolsky DK: Inflammatory bowel disease (1). N Engl J Med 325: 928-937, 1991.

2. Fiocchi C: Inflammatory bowel disease: Etiology and pathogenesis. Gastroenterology 115 182-205, 1998.

3. Loftus EV Jr: Clinical epidemiology of inflammatory bowel disease: Incidence, prevalence, and environmental influences. Gastroenterology 126: 1504-1517, 2004.

4. Rogler G, Andus T, Aschenbrenner E, Vogl D, Falk W, Schölmerich J and Gross V: Alterations of the phenotype of colonic macrophages in inflammatory bowel disease. Eur J Gastroenterol Hepatol 9: 893-899, 1997.

5. Xavier RJ and Podolsky DK: Unravelling the pathogenesis of inflammatory bowel disease. Nature 448: 427-434, 2007.

6. Mowat AM: Anatomical basis of tolerance and immunity to intestinal antigens. Nat Rev Immunol. 3: 331-341, 2003.

7. Rogler G and Andus T: Cytokines in inflammatory bowel disease. World J Surg 22: 382-389, 1998.

8. Banks C, Bateman A, Payne R, Johnson P and Sheron N: Chemokine expression in IBD. Mucosal chemokine expression is unselectively increased in both ulcerative colitis and Crohn's disease. J Pathol 199: 28-35, 2003.

9. Jurjus AR, Khoury NN and Reimund JM: Animal models of inflammatory bowel disease. J Pharmacol Toxicol Methods 50: 81-92, 2004.

10. Garside P: Cytokines in experimental colitis. Clin Exp Immunol 118: 337-339, 1999.

11. Kamada N, Hisamatsu T, Okamoto S, Sato T, Matsuoka K, Arai K, Nakai T, Hasegawa A, Inoue N, Watanabe N, et al: Abnormally differentiated subsets of intestinal macrophage play a key role in Th-1-dominat chronic colitis through excess production of IL-12 and IL-23 in response to bacteria. J Immunol 175: 6900-6908, 2005.

12. Watanabe N, Ikuta K, Okazaki K, Nakase H, Tabata Y, Matsuura M, Tamaki H, Kawanami C, Honjo T and Chiba T: Elimination of local macrophages in intestine prevents chronic colitis in interleukin-10-deficient mice. Dig Dis Sci 48: 408-414, 2003.

13. Hunter MM, Wang A, Parhar KS, Johnston MJ, Van Rooijen N, Beck PL and McKay DM: In vitro-derived alternatively activated macrophages reduce colonic inflammation in mice. Gastroenterology 138: 1395-1405, 2010.

14. Rivollier A,He J,Kole A, Valatas V and Kelsall BL: Inflammation switches the differentiation program of Ly6Chi monocytes from antiinflammatory macrophages to inflammatory dendritic cells in the colon. J Exp Med 209: 139-155, 2012.

15. Gordon S and Taylor PR: Monocyte and macrophage heterogeneity. Nat Rev Immunol 5: 953-964, 2005.
16. Weterman MA, Ajubi N, van Dinter IM, Degen WG, van Muijen GN, Ruitter DJ and Bloemers HP: nmb, a novel gene, is expressed in low-metastatic human melanoma cell lines and xenografts. Int J Cancer 60: 73-81, 1995.

17. Safadi FF, Xu J, Smock SL, Rico MC, Owen TA and Popoff SN: Cloning and characterization of osteoactivin, a novel cDNA expressed in osteoblasts. J Cell Biochem 84: 12-26, 2001.

18. Shikano S, Bonkobara M, Zukas PK and Ariizumi K: Molecular cloning of a dendritic cell-associated transmembrane protein DC-HIL, that promotes RGD-dependent adhesion of endothelial cells through recognition of heparan sulfate proteoglycans. J Biol Chem 276: 8125-8134, 2001 .

19. Onaga M, Ido A, Hasuike S, Uto H, Moriuchi A, Nagata K, Hori T, Hayash $\mathrm{K}$ and Tsubouchi $\mathrm{H}$ : Osteoactivin expressed during cirrhosis development in rats fed a choline-deficient, L-amino acid-defined diet, accelerates motility of hepatoma cells. J Hepatol 39: 779-785, 2003.

20. Abe H, Uto H, Takami Y, Takahama Y, Hasuike S, Kodama M, Nagata K,Moriuchi A, Numata M, Ido A and Tsubochi H: Transgenic expression of osteoactivin in the liver attenuates hapatic fibrosis in mice. Biochem Biophys Res Commun 356: 610-615, 2007.

21. Ripoll VM, Irvine KM, Ravasi T, Sweet MJ and Hume DA: Gpnmb is induced in macrophages by IFN-gammma and lipopolysaccharide and acts as a feedback regulator of proinflammatory responses. J Immunol 178: 6557-6566, 2007.

22. Chung JS, Dougherty I, Cruz PD Jr and Ariizumi K: Syndecan-4 mediates the coinhibitory function of DC-HIL on T cell activation. J Immunol 179: 5778-5784, 2007.

23. Howell GR, Libby RT, Marchant JK, Wilson LA, Cosma IM, Smith RS, Anderson MG and John SW: Absence of glaucoma in DBA/2J mice homozygous for wild-type versions of Gpnmb and Tyrp1. BMC Genetics 8: 45, 2007.

24. Cooper HS, Murthy SN, Shah RS, Sedergran DJ: Clinicopathological study of dextran sulfate sodium experimental murine colitis. Lab Invest 69: 238-249, 1993.

25. Kihara N, de la Fuente SG, Fujino K, Takahashi T, Pappas TN and Mantyh CR: Vanilloid receptor-1 containing primary sensory neurons mediate dextran sulphate sodium induced colitis in rats. Gut 52: 713-719, 2003

26. Mosser DM and Edwards JP. Exploring the full spectrum of macrophage activation. Nat Rev Immunol 8: 958-969, 2008.

27. Ramachandran P,Pellicoro A,Vernon MA,Boulter L, Aucott RL, Ali A, Hartland SN, Snowdon VK, Cappon A, Gordon-Walker TT, et al: Differential Ly-6C expression identifies the recruited macrophage phenotype, which orchestrates the regression of murine liver fibrosis. Proc Natl Acad Sci USA 109: E3186-E3195, 2012.

28. Daley JM, Brancato SK, Thomay AA, Reichner JS and Albina JE: The phenotype of murine wound macrophages. J Leukoc Biol 87: 59-67, 2010.

29. Zhou X, Li F, Kong L, Tomita H, Li C and Cao W: Involvement of inflammation, degradation, and apoptosis in a mouse model of glaucoma. J Biol Chem 280: 31240-31248, 2005

30. Perdiguero E, Sousa-Victor P, Ruiz-Bonilla V, Jardí M, Caelles C, Serrano AL and Muñoz-Cánoves P: p38/MKP-1-regulated AKT coordinates macrophage transitions and resolution of inflammation during tissue repair. J Cell Biol 195: 307-322, 2011.

31. Arranz A, Doxaki C, Vergadi E, Martinez de la Torre Y, Vaporidi K, Lagoudaki ED, Ieronymaki E, Androulidaki A, Venihaki M, Margioris AN, et al: Aktl and Akt2 protein kinases differentially contribute to macrophage polarization. Proc Natl Acad Sci USA 109: 9517-9522, 2012 